03

\title{
Двухфотонное поглощение излучения нецепного HF-лазера в монокристаллах германия
}

\author{
(C) Е.Е. Алексеев ${ }^{1}$, С.Ю. Казанцев ${ }^{2}$, И.Г. Кононов ${ }^{2}$, В.Е. Рогалин ${ }^{3,4}$, К.Н. Фирсов ${ }^{2,5}$ \\ ${ }^{1}$ НИЦ „Курчатовский институт“, \\ 123182 Москва, Россия \\ ${ }^{2}$ Институт общей фризики РАН, \\ 119991 Москва, Россия \\ ${ }^{3}$ НЦЛСК Астрофризика, \\ 420424 Москва, Россия \\ ${ }^{4}$ Тверской государственный университет, \\ 170100 Тверь, Россия \\ ${ }^{5}$ Научно-исследовательский ядерный университет МИФИ, \\ 115409 Москва, Россия \\ e-mail: v-rogalin@mail.ru
}

Поступила в редакцию 15.08.2017 г.

В окончательной редакции 05.02.2018 г.

\begin{abstract}
Представлены результаты экспериментального и численного исследований прохождения излучения нецепного $\mathrm{HF}(\mathrm{DF})$-лазера через монокристаллы германия $(\mathrm{Ge})$ различной толщины и удельного сопротивления. На основе экспериментальных данных для спектра генерации HF(DF)-лазера сделаны оценки коэффициента двухфотонного поглощения в $\mathrm{Ge}-K_{2}=55 \pm 10 \mathrm{~cm} / \mathrm{GW}$ для $\lambda=2.8 \mu \mathrm{m}$. Результаты хорошо согласуются с теорией. Разработанная программа обработки данных эксперимента позволила численно исследовать прохождение мощного излучения с $\lambda=2.6-3 \mu \mathrm{m}$ через $\mathrm{Ge}$ в любой момент воздействия лазерного импульса. Показано, что для мощного лазерного излучения с $\lambda=2.6-4 \mu \mathrm{m}$ тонкие покрытия из Ge могут эффективно выравнивать распределение энергии по апертуре пучка.
\end{abstract}

DOI: $10.21883 /$ OS.2018.06.46082.180-17

\section{Введение}

Германий, будучи наиболее исследованным полупроводниковым монокристаллом, активно используется не только в электронной промышленности, но и в ИК оптике. В настоящее время это наиболее технологически чистый из исследованных материалов и наиболее изученный. Диапазон прозрачности германия $1.8-23 \mu \mathrm{m}$, однако он прозрачен и в терагерцовом диапазоне. Этот материал обладает высоким показателем преломления $\sim 4.0$ и хорошими термомеханическими и климатическими свойствами и, что важно для ИК приборов, не прозрачен в видимой области [1].

В ИК области два окна прозрачности атмосферы $3-5$ и 8-14 $\mu \mathrm{m}$. Для этих диапазонов создано большое количество приборов (в том числе и лазерных), использующих оптику из германия [2-6]. Среди применений германия в ИК оптике следует отметить окна и объективы тепловизионных приборов, входные окна фотоприемников [6]. Эти изделия зачастую эксплуатируются совместно с различными лазерными приборами. В области 2.6-4.2 $\mu \mathrm{m}$ наиболее мощные источники излучения - это $\mathrm{HF}(\mathrm{DF})$-лазеры $[2,7-10]$, в диапазоне 4-8 $\mu \mathrm{m}$ - это СО-лазеры [11], а в $8-14 \mu \mathrm{m}-$ это $\mathrm{CO}_{2-}$ и $\mathrm{N}_{2} \mathrm{O}$-лазеры [12]. В настоящее время также ведутся активные работы по созданию мощных импульсно-периодических твердотельных лазеров, излучающих в спектральном диапазоне 3-5 $\mu \mathrm{m}$ [13-16].
Появление достаточно мощных и доступных в ближайшей перспективе лазерных устройств, излучающих в спектральной области среднего ИК диапазона, обусловливает актуальность исследования взаимодействия мощного лазерного излучения с германием. Изученность германия также способствует более глубокому пониманию физики процесса и должно помогать правильной эксплуатации этого материала в разнообразных ИК приборах.

Германий $(\mathrm{Ge})$ - полупроводник с шириной запрещенной зоны $E_{g}=0.67 \mathrm{eV}$, что способствует возникновению различных нелинейных эффектов при взаимодействии излучения с материалом. Для лазерной оптики основной эффект - нелинейное поглощение на генерированных излучением неравновесных носителях заряда $[3,5]$. В области 2.6-5 $\mu$ m на этот процесс накладываются эффекты двухфотонного [17-25] и трехфотонного поглощений [23]. В эксперименте эти процессы разделить крайне трудно, кроме этого, результаты исследований могут существенно зависеть от таких параметров лазерного источника, как длина волны, распределение энергии по апертуре лазерного пучка и размеров луча на кристалле Ge. Поэтому корректное измерение коэффициентов поглощения для каждого процесса является сложной проблемой, и не удивительно, что величина коэффициента двухфотонного поглощения $K_{2}$ в различных работах существенно отличается (в пределах двух порядков) [17-25]. 
В настоящей работе проведены экспериментальное и численное исследования этого явления с использованием излучения нецепного импульсного НF-лазера с высоким качеством лазерного пучка [26]. Влияние длины волны излучения - $\lambda$ проверено заменой активной среды HF на DF, что позволило оценить разницу между двухфотонными и трехфотонными явлениями.

\section{Экспериментальная установка и методика исследований}

Нелинейное поглощение исследовалось на образцах $\mathrm{Ge}$ различной толщины и удельного сопротивления. Рассмотрено влияние дислокационной структуры германия.

Для выявления динамики поглощения была разработана компьютерная программа для вычисления мгновенных значений интенсивности прошедшего излучения и концентрации генерируемых в $\mathrm{Ge}$ свободных носителей.

Исследовались оптически обработанные плоскопараллельные пластины $\mathrm{Ge}$ (марки ГМО) $n$-типа с удельным сопротивлением $\rho \sim 2-40 \Omega \cdot \mathrm{cm}$ различной толщины $h=0.3-5.5 \mathrm{~mm}$ со стандартной концентрацией дислокаций $\sim 10^{5} \mathrm{~cm}^{-2}$ и образцы бездислокационного Ge $h=0.3 \mathrm{~mm}$. Для сопоставления исследованы пластины других полупроводников - кремния $\left(\mathrm{Si} ; E_{g}=1.12\right)$ и арсенида галлия $\left(\mathrm{GaAs} ; E_{g}=1.42\right)$ оптического качества, $h=6 \mathrm{~mm}$.

Источником излучения служил нецепной $\mathrm{HF}(\mathrm{DF})$-лазер, описанный в работах [26,27]. Длительность лазерного импульса по полувысоте составляла $\tau \sim 150 \mathrm{~ns}$; максимальная энергия $E \approx 5 \mathrm{~J}$. В зависимости от активной среды лазер генерировал излучение $2.7-3 \mu \mathrm{m}$ (НF-лазер), либо в 3.6-4.1 $\mu \mathrm{m}$ (DF-лазер) [28]. Спектр генерации лазера был аналогичен тому, что приведен в [28].

Схема экспериментов показана на рис. 1. Излучение лазера фокусировалось на $\mathrm{Ge}$ сферической линзой из $\mathrm{BaF}_{2}$ с фокусным расстоянием $L=45 \mathrm{~cm}$. Калиброванными ослабителями из тефлона $F$ последовательно изменялась плотность энергии на $\mathrm{Ge}$ в диапазоне $W_{\text {in }}=0.01-3 \mathrm{~J} / \mathrm{cm}^{2}$, ирисовой диафрагмой $D$ регулировался диаметр пятна облучения на кристалле $\mathrm{Ge}$

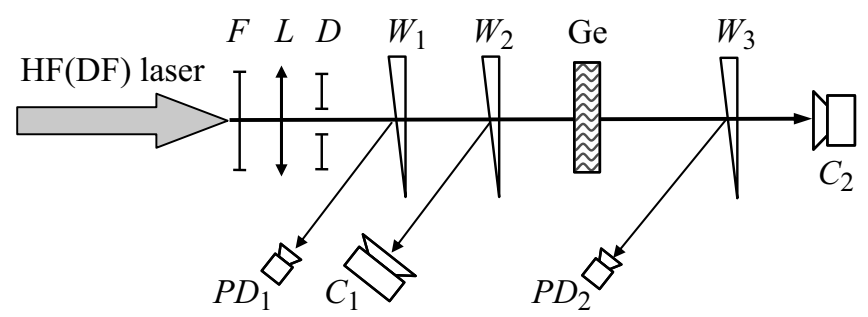

Рис. 1. Схема экспериментальной установки: $F-$ набор калиброванных ослабителей; $L-$ сферическая линза; $D-$ ирисовая диафрагма; $W_{1}, W_{2}, W_{3}-$ оптические клинья из $\mathrm{CaF}_{2}$; $C_{1}, C_{2}-$ калориметры для измерения энергии излучения; $P D_{1}, P D_{2}$ - фотоприемники. $d_{\text {in }}=2-9 \mathrm{~mm}$ (это позволяло учесть размерный эффект). Энергии воздействующего излучения $\left(E_{\text {in }}\right)$, прошедшего через образец $\left(E_{\mathrm{tr}}\right)$, измерялись калориметрами $C_{1}$ и $C_{2}$ фирмы Gentec соответственно.

Форма импульса излучения, падающего и прошедшего через $\mathrm{Ge}$, контролировалась фотоприемниками $\left(P D_{1}\right.$, $\left.P D_{2}\right)$ фирмы Vigo-system Ltd с временным разрешением $\sim 1.5 \mathrm{~ns}$. Сигналы с фотоприемников регистрировались осциллографом Tektronix 2012B с полосой пропускания $100 \mathrm{MHz}$.

\section{Экспериментальные результаты и обсуждение}

В исследованном диапазоне $W_{\text {in }}=0.01-3 \mathrm{~J} / \mathrm{cm}^{2}\left(I_{\text {las }}=\right.$ $\left.=0.02-20 \mathrm{MW} / \mathrm{cm}^{2}\right)$ повреждений поверхности $\mathrm{Ge}$ не наблюдалось, т. е. порог оптической стойкости материала не достигался. Однако уже при достаточно низких интенсивностях $\left(I_{\text {las }} \geq 0.3 \mathrm{MW} / \mathrm{cm}^{2}\right)$ облучения наблюдался существенно нелинейный характер прохождения излучения через Ge. При исследовании зависимостей пропускания (параметра $T=E_{\mathrm{tr}} / E_{\text {in }}$ ) от плотности падающего на кристалл излучения HF-лазера для кристаллов $\mathrm{Ge}$, арсенида галлия и кремния толщиной $6 \mathrm{~mm}$ обнаружено, что на образцах $\mathrm{GaAs}$ и $\mathrm{Si}$ в исследуемом диапазоне $I_{\text {las }}=0.01-20 \mathrm{MW} / \mathrm{cm}^{2}$ нелинейное поглощение не наблюдалось, и величина пропускания $T$ определялась только потерями на френелевское отражение. Пропускание Ge при $I_{\text {las }} \geq 3 \mathrm{MW} / \mathrm{cm}^{2}$ заметно уменьшается. Исследования пропускания Ge различной толщины $(h)$ и удельного сопротивления $(\rho)$ показали, что в области нелинейности $T$ заметно уменьшается при увеличении толщины образца и уменьшении удельного сопротивления кристалла.

При исследовании зависимости плотности прошедшей энергии от плотности, падающей на $\mathrm{Ge}$ энергии HF-лазера, полученной при диаметрах облученной зоны соответственно 7.4 и $2.7 \mathrm{~mm}$, обнаружено, что при достаточно высоких плотностях облучения пропускание при малых пятнах выше, чем при больших. Этот размерный эффект наблюдался во всех образцах Ge.

На рис. 2 видно, что не только мощность прошедшего импульса заметно уменьшается, но и его форма сильно искажается, передний фронт заметно укорачивается. Длительность $t_{m}$ переднего фронта импульса, прошедшего через пластинку германия (измерялся от начала импульса генерации до момента времени, когда прошедший импульс достигает максимума), от значения мощности падающего излучения (в точке $t_{m}$ ) уменьшается при росте интенсивности излучения падающего на кристалл.

Интенсивность излучения HF-лазера, прошедшего через $\mathrm{Ge}$, достигает максимума раньше, чем интенсивность падающего импульса и тем быстрее, чем выше интенсивность. Это свидетельствует, что основной вклад в нелинейность пропускания $\mathrm{Ge}$ дает поглощение на неравновесных носителях заряда, генерированных 


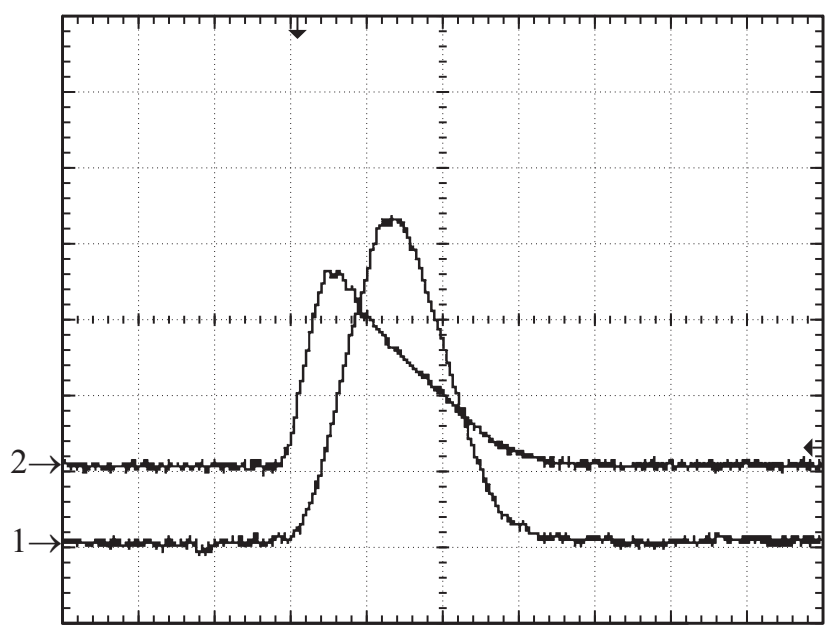

Рис. 2. Осциллограммы импульсов излучения НF-лазера: канал 1 - падающий на кристалл $\mathrm{Ge}$ (калибровка $1.4 \mathrm{MW} / \mathrm{div}) ;$ канал 2 - прошедший через кристалл (калибровка $138 \mathrm{~kW} / \mathrm{div})$, развертка $100 \mathrm{~ns} / \mathrm{div}$. Получено на образце бездислокационного $\mathrm{Ge}$ толщиной $300 \mu \mathrm{m}$ при $E_{\mathrm{in}}=0.918 \mathrm{~J}$ и диаметре пятна облучения $d_{\text {in }}=6.5 \mathrm{~mm}\left(W_{\text {in }}=2.8 \mathrm{~J} / \mathrm{cm}^{2}\right)$.

в объеме кристалла германия за счет двухфотонного поглощения излучения HF-лазера [17-25].

В [17] на основе этого предположения получено асимптотическое соотношение (1), которое связывает интенсивности прошедшего и падающего на кристалл излучения HF-лазера в области достаточно высоких плотностей облучения:

$$
\left(\frac{1}{T^{2}}-1\right)^{1 / 2}=\left(\frac{K_{2} \tau_{\mathrm{las}} Z\left(\sigma_{p}+\sigma_{e}\right)}{\eta \hbar \omega}\right)^{1 / 2} I_{0} .
$$

Здесь $T=I_{z} / I_{0} ; I_{z}$ - интенсивность излучения, прошедшего слой кристалла толщиной $Z ; I_{0}-$ интенсивность излучения входящего в кристалл; $K_{2}-$ коэффициент двухфотонного поглощения в германии; $\tau_{\text {las }}$ - длительность лазерного импульса; $Z$ - толщина кристалла германия; $\sigma_{p}, \sigma_{e}-$ сечение поглощения фотонов HFлазера соответственно свободными дырками и электронами; $\eta$ - некий коэффициент, зависящий от формы лазерного импульса; $\hbar \omega-$ энергия лазерного фотона.

На основе полученных экспериментальных данных были построены зависимости $\left(1 / T^{2}-1\right)^{1 / 2}$ от $I_{0}$. Установлено, что для всех исследованных образцов Ge параметр $\left(1 / T^{2}-1\right)^{1 / 2}$ линейно зависит от интенсивности падающего излучения: $\left(1 / T^{2}-1\right)^{1 / 2}=k_{g} I_{0}+A$. Величина $k_{g}$ растет с увеличением толщины образца $\left(k_{g} \sim \sqrt{h}\right)$.

Однако непосредственное определение $K_{2}$ из экспериментальных зависимостей затруднительно из-за неопределенности ряда факторов. Например, как отмечено в [17], форма лазерного импульса и распределение по пятну заметно влияют на численное значение рассчитанного коэффициента $K_{2}$. Поэтому была разработана компьютерная программа для получения расчетным путем мгновенных значений интенсивности и коэффициентов поглощения излучения HF-лазера в $\mathrm{Ge}$. Для определения значений коэффициента $K_{2}$ в программе учитывались реальные форма импульса и распределение излучения по апертуре пятна.

Система уравнений (2), (3), описывающая изменение интенсивности излучения $I$ при прохождении $\mathrm{Ge}$ и концентрации свободных носителей $\Delta N$ [17]:

$$
\begin{gathered}
-\frac{d I}{d z}=K_{1} I+K_{2} I^{2}+\left(\sigma_{e}+\sigma_{p}\right) \Delta N I, \\
\frac{d \Delta N}{d t}=\frac{K_{2} I^{2}}{2 \hbar \omega}-\beta \Delta N,
\end{gathered}
$$

$I(z, t, r)$ - локальная интенсивность излучения в $\mathrm{Ge}$ (в момент времени $t$ ) на глубине $z$, на расстоянии $r$ от оси пучка (предполагалось, что распределение интенсивности по пятну аксиально симметрично). Ось $z$ совпадает с направлением распространения лазерного излучения, а начало координат с входной плоскостью образца. $K_{1}$ - это линейный коэффициент поглощения на свободных носителях в $\mathrm{Ge}$.

Моделирование проводилось для средней $\lambda=2.82 \mu \mathrm{m}$ (усреднение проводилось с учетом спектрального распределения излучения нецепного HF-лазера, использовавшегося в экспериментах).

При расчете учитывалась реальная форма импульса и распределение интенсивности по пятну, а нагревом свободных носителей и диффузией свободных носителей пренебрегали, поскольку длительность воздействующего импульса не превышала $300 \mathrm{~ns}$.

Плотность распределения энергии излучения, воздействующего на $\mathrm{Ge}$, аппроксимировалась функцией Гаусса $\Phi(r)=S / \pi \delta \exp \left(-r^{2} / \delta\right)$. В программе осуществлялось варьирование параметров модели $K_{2}$ и $K_{1}$ так, чтобы обеспечить максимальное совпадение расчетного им-

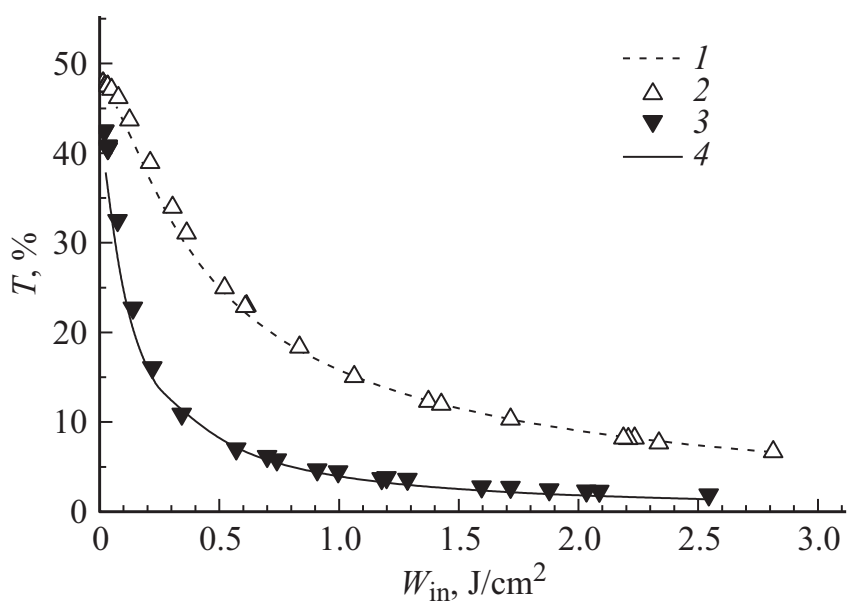

Рис. 3. Экспериментальные и расчетные зависимости пропускания кристаллов разной толщины от плотности энергии излучения HF-лазера: 1 - расчет для $h=0.3 \mathrm{~mm} ; 2-$ эксперимент для $h=0.3 \mathrm{~mm} ; 3-$ эксперимент, $h=5.5 \mathrm{~mm}$; 4 - расчет для кристалла Ge толщиной $h=5.5 \mathrm{~mm}$. Диаметр облученной зоны $d_{\text {in }}=6.4 \mathrm{~mm}$. 

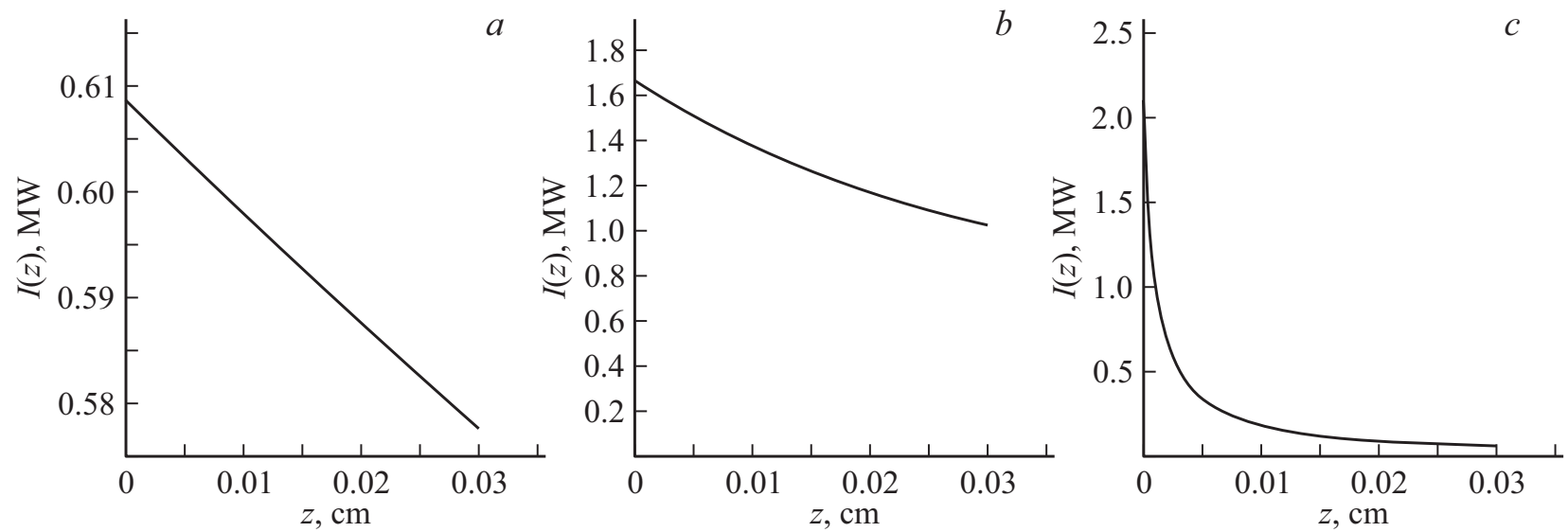

Рис. 4. Распределение интенсивности излучения НF-лазера по глубине кристалла Gе в моменты времени $t \approx 20 \mathrm{~ns}(a), \approx 60 \mathrm{~ns}(b)$, $\approx 200 \mathrm{~ns}(c)$ от начала лазерного импульса.

пульса, прошедшего слой $\mathrm{Ge}$ толщиной $h$, и экспериментального. Расчет проводился при следующих значениях параметров: $K_{1}=0.003 \mathrm{~cm}^{-1}, K_{2}=55 \mathrm{~cm} / \mathrm{GW}$, $\sigma_{e}=0.72 \cdot 10^{-17} \mathrm{~cm}^{2}, \sigma_{p}=1.44 \cdot 10^{-16} \mathrm{~cm}^{2}, \tau_{\mathrm{Ge}}=1 \mathrm{~ms}$, $h=0.03 \mathrm{~cm}, E_{\text {in }}=0.92 \mathrm{~J}, d_{\text {in }}=6.5 \mathrm{~mm}, S=0.33 \mathrm{~cm}^{2}$.

Наилучшее согласие результатов численного моделирования и эксперимента для образцов толщиной $h=0.03-0.55 \mathrm{~cm}$ (рис. 3) достигалось при значениях $K_{2}=55 \pm 10 \mathrm{~cm} / \mathrm{GW}$. Как видно на рис. 4, сгенерированные носители сосредоточены в тонком слое вблизи входной поверхности $\mathrm{Ge}$, т.е. основные процессы нелинейного поглощения происходят в узком слое толщиной менее $50 \mu \mathrm{m}[29,30]$. Таким образом, генерация неравновесных носителей в этом слое ограничивает прохождение лазерного излучения величиной некой предельной интенсивности. Возникший нелинейный фильтр препятствует разрушению материала даже при попытке жестко сфокусировать луч в глубину кристалла.

Численное моделирование также выявило основную причину обнаруженного в эксперименте размерного эффекта (рост пропускания при увеличения размера пятна облучения). Обычно, когда на краю пятна плотность лазерной энергии падает более чем на порядок, исследователи не учитывают наличие небольшого пьедестала на распределении плотности энергии по пятну. С ростом радиуса пятна облучения доля энергии, заключенная во внешнем кольцевом слое, растет, а поскольку плотность энергии в этом слое недостаточна для генерации свободных носителей, то эта часть падающей энергии свободно проходит через кристалл германия, практически не поглощаясь.

\section{Заключение}

Проведены экспериментальные исследования прохождения излучения HF(DF)-лазера через Ge. Разработаны численная модель и компьютерная программа, которая с хорошей точностью не только описывает результаты эксперимента, но и позволяет рассмотреть динамику процесса в любой заданный момент времени в течение лазерного импульса.

На основании анализа результатов эксперимента путем численного моделирования процесса поглощения излучения определена эффективная по спектру генерации HF-лазера $(\lambda=2.82 \mu \mathrm{m})$ величина коэффициента двухфотонного поглощения $K_{2}=55 \pm 10 \mathrm{~cm} / \mathrm{GW}$.

Полученные данные могут быть использованы для более глубокого понимания процессов нелинейного поглощения в $\mathrm{Ge}$ с целью практического применения, например при создании эффективных ограничителей мощности лазерного излучения диапазона $2.5-4 \mu \mathrm{m}$, а также для выравнивания распределения мощного лазерного излучения после прохождения излучения через слой германия.

Исследования выполнялись в рамках государственного задания по теме „Мощные газовые и твердотельные лазеры ИК диапазона спектра и взаимодействие их излучения с веществом“ АААА-А18-118021690024-6. Работа была также частично поддержана грантом РФФИ № 18-08-00793 в части разработки экспериментальных методик и проведения лазерных исследований.

\section{Список литературы}

[1] Каплунов И.А., Колесников А.И., Гавалян М.Ю., Белоцерковский А.В. // Опт. и спектр. 2016. Т. 120. № 4. C. 691-696. doi 10.7868/S0030403416030132; Kaplunov I.A., Kolesnikov A.I., Gavalyan M.Yu., Belotserkovskiy A.V. // Opt. Spectrosc. 2016. V. 120. N 4. P. 654-659. doi 10.1134/S0030400X16030139

[2] Матвиенко Г.Г., Пташник И.В., Романовский О.А. и др. // Прикладная физика. 2002. № 1. С. 129-136.

[3] Левинзон Д.И., Ровинский Р.Е., Рогалин В.Е. и др. // Изв. АН СССР (сер. физ.). 1979. Т. 43. № 9. С. 2001-2005.

[4] Аксенов Ю.Н., Борисов В.П., Бурщев В.В. и др. // Квант. электрон. 2001. Т. 31. № 4. С. 290-292; Aksenov Yu.N., Borisov V.P., Burtsev V.V. et al. // Quant. Electron. 2001. V. 31. N 4. P. $290-292$. 
[5] Ровинский Р.Е., Рогалин В.Е., Шершель В.А. // Изв. АН СССР (сер. физ.). 1983. Т. 47. № 2. С. 406-409.

[6] Коротаев В.В., Мельников Г.С., Михеев С.В., Самков В.М., Солдатов Ю.И. Основы тепловидения. СПб.: ИТМО, 2012. $123 \mathrm{c}$.

[7] Apollonov V.V., Belevtsev A.A., Firsov K.N. et al. // Proc. SPIE. 2002. V. 4747. P. 31-43. doi 10.1117/12.460146

[8] Булаев В.Д., Гусев В.С., Казанцев С.Ю. и др. // Квант. электрон. 2010. Т. 40. № 7. С. 615-618; Bulaev V.D., Gusev V.S., Kazantsev S.Yu. et al. // Quant. Electron. 2010. V. 40. N 7. P. $615-618$. doi 10.1070/QE2010v040n07ABEH014323

[9] Великанов С.Д., Домажиров А.П., Зарецкий Н.А. и др. // Квант. электрон. 2015. Т. 45. № 11. С. 989-992; Velikanov S.D., Domazhirov A.P., Zareckij N.A. et al. // Quant. Electron. 2015. V. 45. N 11. P. 989-992. doi 10.1070/QE2015v045n11ABEH015889

[10] Великанов С.Д., Гаранин С.Г., Домажсиров А.П. и др. // Квант. электрон. 2010. Т. 40. № 5. С. 393-396; Velikanov S.D., Garanin S.G., Domazhirov A.P. et al. // Quant. Electron. 2010. T. 40. N 5. C. 393-396. doi 10.1070/QE2010v040n05ABEH014324

[11] Андреев Ю.М., Ионин А.А., Киняевский И.О. и др. // Квант. электрон. 2013. Т. 43. № 2. С. 139-143; Andreev Yu.M., Ionin A.A., Kinyaevsky I.O. et al. // Quant. Electron. 2013. V. 43. N 2. P. 139-143; doi 10.1070/QE2013v043n02ABEH014978

[12] Аполлонов В.В., Васьковский Ю.М., Жаворонков М.И. и дp. // Квант. электрон. 1985. Т. 12. № 1. С. 5-9; Apollonov V.V., Vas'kovskiu Yu.M., Zhavoronkov M.I. et al. // Soviet J. Quant. Electron. 1985. V. 15. N 1. P. 1-3. doi 10.1070/QE1985v015n01ABEH005831

[13] Dormidonov A.E., Firsov K.N., Gavrishchuk E.M. et al. // Appl. Phys. B. 2016. V. 122-211. doi 10.1007/s00340-0166489-6

[14] Великанов С.Д., Гаврищук Е.М., Зарецкий Н.А. и дp. // Квант. электрон. 2017. Т. 47. № 4. С. 303-307; Velikanov S.D., Gavrishchuk E.M., Zareckij N.A. et al. // Quant. Electron. 2017. V. 47. N 4. P. 303-307. doi 10.1070/QEL16324

[15] Firsov K.N., Gavrishchuk E.M., Ikonnikov V.B et al. // Laser Phys. Lett. 2016. V. 13. P. 065003. doi 10.1088/16122011/13/6/065003

[16] Firsov K.N., Gavrishchuk E.M., Ikonnikov V.B., et al. // Laser Phys. Lett. 2017. V. 14. P. 055805. doi 10.1088/1612-202X/aa66fb

[17] Gibson A., Hatch C., Maggs P. et al. // J. Phys. C: Chern. Solid State Phys. 1976. V. 9. P. 3259-3275.

[18] Зубов Б.В., Кулевский Л.А., Макаров В.П. и др. // Письма ЖЭТФ. 1969. Т. 9. № 4. С. 221-224.

[19] Зубов Б.В., Мурина Т.М., Оловянин Б.Р., Прохоров А.М. // ФТП. 1971. Т. 5. № 4. С. 636-640.

[20] Wenzel R.G., Arnold G.P., Greiner N.R. // Appl. Opt. 1973. V. 12. P. $2245-6$.

[21] Garcia H., Avanaki K.N. // Appl. Phys. Lett. 2012. V. 100. P. 131105. doi org/10.1063/1.3693389

[22] Wagner T.J., Bohn M.J., Coutu A Jr.R. et al. // JOSA B. 2010. V. 27. N 10. P. $2122-2131$.

[23] Seo D., Gregory J.M., Feldman L.C. et al. // Phys. Rev. B. 2011. V. 83. P. 195203.

[24] Rauscher C., Laenen R. // J. Appl. Phys. 1997. V. 81. P. 2818.

[25] Сидорин А.В. // Труды ФИАН. Т. 137 / Под ред. Басова Н.Г. М.: Наука, 1982. С. 135-190.
[26] Игнатьев А.Б., Казанцев С.Ю., Кононов И.Г. и др. // Квант. электрон. 2008. Т. 38. № 1. С. 69-72; Ignat'ev A.B., Kazantsev S.Yu., Kononov I.G. et al. // Quant. Electron. 2008. T. 38. N 1. C. $69-72$. doi 10.1070/QE2008v038n01ABEH013546

[27] Казанщев С.Ю., Кононов И.Г., Коссый И.А., Тарасова Н.М., Фирсов К.Н. // Физика плазмы. 2009 Т. 35. № 3. C. 281-288; Kazantsev S.Yu., Kononov I.G., Kossyi I.A. et al. // Plasma Phys. Rep. 2009. V. 35. N 3. P. 251-257. doi 10.1134/s1063780x09030088

[28] Firsov K.N., Kazantsev S.Yu., Kononov I.G. et al. // Laser Phys. Lett. 2014. V. 11. N 12. P. 125004. doi 10.1088/16122011/11/12/125004

[29] Рогалин B.E. // Известия вузов. Материалы электронной техники. 2013. № 2. С. 11-18.

[30] Каплунов И.А., Рогалин В.Е., Гавалян М.Ю. // Опт. и спектр. 2015. Т. 118. № 2. С. 254-260. doi 10.7868/S0030403415020087; Kaplunov I.A., Rogalin V.E., Gavalyan M.Yu. // Opt. Spectrosc. 2015. V. 118. N 2. P. 240-246. doi 10.1134/S0030400X15020083 